\title{
REPRESENTATION OF FUNDAMENTAL SOLUTIONS FOR GENERALIZED CAUCHY-RIEMANN EQUATIONS BY QUASICONFORMAL MAPPINGS
}

\author{
M. SCHIFFER and G. SCHOBER
}

\section{Introduction}

Let $D$ be a domain in the extended complex plane $\bar{C}=C \cup\{\infty\}$, and let $K: D \rightarrow[1, \infty)$ be a measurable function. We shall assume that

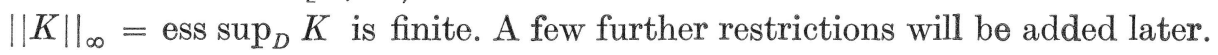

Definition. A homeomorphism $f: D \rightarrow \bar{C}$ is $K(z)$-quasiconformal ( $K(z)$-q.c.) if $f$ is locally absolutely continuous on a.e. horizontal and vertical line (ACL) in $D$ and satisfies the dilatation condition

$$
1 \leqq \frac{\left|f_{z}(z)\right|+\left|f_{\bar{z}}(z)\right|}{\left|f_{\bar{z}}(z)\right|-\left|f_{\bar{z}}(z)\right|} \leqq K(z) \quad \text { for a.e. } z \in D
$$

Since $\|K\|_{\infty}<\infty$, each $K(z)$-q.c. mapping is $\|K\|_{\infty}$-quasiconformal according to the standard definitions ([2]). If $\infty \in D$, we shall require that $f(\infty)=\infty$; otherwise $f(D) \subset C$ if $\infty \notin D$. The family of all such $K(z)$-q.c. mappings of $D$ is denoted by $Q(K, D)$, and we abbreviate $Q(K)=$ $Q(K, \bar{C})$.

We shall pose some extremal problems concerned with the global distortion of $K(z)$-q.c. mappings. Their resolution by variational methods will provide weak fundamental solutions to the partial differential equations

$$
\operatorname{div}(K \operatorname{grad} V)=0 \quad \text { and } \quad \operatorname{div}((1 / K) \operatorname{grad} V)=0 .
$$

These equations are, of course, of basic importance in the theory of steady state heat flow (fluid flow, electrostatics) in an inhomogeneous medium. Our methods will simultaneously yield the existence of fundamental solutions for (1.2) and a representation for them in terms of $K(z)$-q.c.

This work was supported in part by NSF grants GP35543 and MPS 75-07123.

doi:10.5186/aasfm.1976.0232 
mappings. It is the representation in terms of univalent functions that is for us the principal attraction of the theory.

By weak solution of the equation $\operatorname{div}(K \operatorname{grad} V)=0$ we mean a continuous function $V$ that admits locally a continuous conjugate $U$ in the sense that $U$ and $V$ are locally ACL and their first order partial derivatives are locally in $L^{2}$ and satisfy the generalized Cauchy-Riemann equations

$$
U_{x}=K V_{y}, \quad U_{y}=-K V_{x} .
$$

The theory of the system

$$
U_{x}=K V_{y}, \quad U_{y}=-\frac{1}{K} V_{x}
$$

is well known in terms of q.c. mappings. For if we define $F=U+i V$, then (1.4) is equivalent to the Beltrami equation

$$
F_{z}=\frac{K-1}{K+1} F_{z} .
$$

Generalized solutions of (1.5) are well known (e.g. [2]) to be expressible as the composition of a $K(z)$-q.c. mapping with any analytic function. On the other hand, the system (1.3) is equivalent to the equation

$$
F_{\bar{z}}=\frac{K-1}{K+1} \overline{F_{z}} .
$$

It is a feature of the variational method that it leads to solutions of this latter equation.

Very briefly, the variational method $[1,3,6,7,9]$ leads to the following procedure: Let $\chi[f]$ be a functional that admits the following asymptotic formula

$$
\chi[f+\varepsilon h]=\chi[f]+\varepsilon \operatorname{Re} L[h ; f]+o(\varepsilon),
$$

where $L$ is a linear functional of $h$, depending also on $f$. Define

$$
\mathfrak{l}(w ; f)=L\left[\frac{1}{f-w} ; f\right]
$$

in many circumstances this will be an analytic function of $w$. If $f$ is an extremal function for the problem of maximizing the functional $\chi$ over a competing family $\mathfrak{\Im}$ of $K(z)$-q.c. mappings, then (under appropriate conditions on $\mathfrak{U}(w ; f))$ it satisfies the differential equation

$$
f_{\bar{z}}(z)=\frac{K(z)-1}{K(z)+1} \frac{|\mathfrak{M}(f(z) ; f)|}{\mathfrak{H}(f(z) ; f)} \overline{f_{z}(z)}
$$


and

$$
F(z)=\int^{f(z)} \sqrt{\mathfrak{u}(w ; f)} d w
$$

satisfies the differential equation (1.6).

The variational formalism makes it evident that we will be particularly successful in extremum problems for which $\mathfrak{U}(w ; f)$ will be the square of some rational function of $w$. This is the same situation as in the theory of conformal mapping. In that theory numerous functionals of this character have been studied and have led to important inequalities. To study the same functionals in the q.c. case is often impossible since the classical functionals depend frequently on the values of the derivative of the mapping function.

In the first sections of this paper, we shall introduce certain functionals of a q.c. mapping, which will have the same functional derivative as the derivative of an analytic function and can be used to reconstruct the classical functionals for q.c. applications. Then we shall use these functionals to obtain existence proofs, to characterize and estimate various Green's functions, and, in particular, to generalize well-known inequalities of Golusin from conformal to very general q.c. mappings.

Our results contain as very special cases some problems in the theory of univalent analytic functions with $K$-q.c. extensions.

\section{Basic functionals}

In order to measure the global distortion of $K(z)$-q.c. mappings, we shall use the expressions

$$
\Phi(z, \zeta ; K, f)=\frac{|f(z)-f(\zeta)|}{|z-\zeta|^{[1 / K(z)+1 / K(\zeta)] / 2}}
$$

and

$$
\Psi(z, \zeta ; K, f)=\frac{|f(z)-f(\zeta)|}{|z-\zeta|^{[K(z)+K(\zeta)] / 2}}
$$

which are defined for $z \neq \zeta$. We shall define $\Phi$ and $\Psi$ also when $z=\zeta$, but only at points where $K$ satisfies an additional condition.

$K$ is essentially continuous at $z_{0}$ if the essential modulus of continuity

$$
\omega\left(r ; z_{0}, K\right)=\operatorname{ess~sup~}_{\left|z-z_{0}\right| \leq r}\left|K(z)-K\left(z_{0}\right)\right|
$$


decreases to zero as $r \rightarrow 0$. We shall impose the additional (Dini) growth restriction that

$$
\int_{0}^{\delta}\left(\omega\left(r ; z_{0}, K\right) / r\right) d r<\infty
$$

for some $\delta>0$. In particular, (2.4) holds if $K$ is just Hölder continuous at $z_{0}$.

Let $A\left(r ; z_{0}, f\right)$ denote the area of $f\left(\left|z-z_{0}\right|<r\right)$. If $K$ is essentially continuous and satisfies $(2.4)$ at $z_{0}$, we define

$$
\Phi\left(z_{0}, z_{0} ; K, f\right)=\lim _{r \rightarrow 0} \frac{\left(A\left(r ; z_{0}, f\right) / \pi\right)^{1 / 2}}{r^{1 / K\left(z_{0}\right)}}
$$

and

$$
\begin{aligned}
\Psi\left(z_{0}, z_{0} ; K, f\right) & =\left[\Phi\left(f\left(z_{0}\right), f\left(z_{0}\right) ; K \circ f^{-1}, f^{-1}\right)\right]^{-K\left(z_{0}\right)} \\
& =\lim _{R \rightarrow 0} \frac{R}{\left[\left(A\left(R ; f\left(z_{0}\right), f^{-1}\right) / \pi\right)^{1 / 2}\right]^{K\left(z_{0}\right)}} .
\end{aligned}
$$

The existence of a (finite) limit in (2.5) and a (possibly infinite) limit in (2.6) will be a consequence of Lemma 1.

The definitions of the functionals $\Phi$ and $\Psi$ are motivated by the fact that q.c. mappings are bi-Hölder-continuous ([2]). For example, if $f \in Q(K, D)$, then for $\alpha=1 /\|K\|_{\infty}$

$$
c_{E}|z-\zeta|^{1 / \alpha} \leqq|f(z)-f(\zeta)| \leqq C_{E}|z-\zeta|^{\alpha}, \quad 0<c_{E} \leqq C_{E}<\infty,
$$

uniformly on each compact subset $E$ of $D$.

In general, the limits

$$
\lim _{z \rightarrow z_{0}} \frac{\left|f(z)-f\left(z_{0}\right)\right|}{\left|z-z_{0}\right|^{1 / K\left(z_{0}\right)}} \text { and } \lim _{z \rightarrow z_{0}} \frac{\left|f(z)-f\left(z_{0}\right)\right|}{\left|z-z_{0}\right|^{K\left(z_{0}\right)}}
$$

need not exist; however, if either does, it is easy to show that it agrees with the corresponding functional (2.5) or (2.6). In any case, (2.5) and (2.6) distinguish limiting points of the quotients in (2.8), that behave well (semicontinuously) as $f$ varies.

\section{Existence and semicontinuity of $\Phi\left(z_{0}, z_{0} ; K, f\right)$ and $\Psi\left(z_{0}, z_{0} ; K, f\right)$}

L e m m a 1. Suppose $f$ is K(z)-q.c. in a neighborhood of $z_{0}$ and $K$ satisfies (2.4) at $z_{0}$. Then 
$B\left(r ; z_{0}, K, f\right) \equiv r^{-2 / K\left(z_{0}\right)} A\left(r ; z_{0}, f\right) \exp \left\{2 K\left(z_{0}\right)^{-2} \int_{0}^{r}\left(\omega\left(\varrho ; z_{0}, K\right) / \varrho\right) d \varrho\right\}$ is nonincreasing as $r \rightarrow 0$. In particular,

$$
\lim _{r \rightarrow 0} r^{-2 / K\left(z_{0}\right)} A\left(r ; z_{0}, f\right)=\lim _{r \rightarrow 0} B\left(r ; z_{0}, K, f\right)
$$

exists.

Proof. Let $J_{f}=\left|f_{z}\right|^{2}-\left|f_{\bar{z}}\right|^{2}$ be the Jacobian of $f$. Then we may represent ([2])

$$
A(r) \equiv A\left(r ; z_{0}, f\right)=\int_{0}^{r} \int_{0}^{2 \pi} J_{f}\left(z_{0}+\varrho e^{i \vartheta}\right) \varrho d \vartheta d \varrho .
$$

As a function of $r, A(r)$ is absolutely continuous, and

$$
A^{\prime}(r)=\int_{0}^{2 \pi} J_{f} r d \vartheta
$$

for a.e. $r$. Since $f$ is q.c., the length $l(r)$ of $f\left(\left|z-z_{0}\right|=r\right)$ is finite for a.e. $r$, and

$$
l(r)=\int_{\left|z-z_{0}\right|=r}|d f|
$$

for a.e. $r$. It follows from (1.1) that

$$
|d f|^{2} \leqq K J_{f}(r d \vartheta)^{2},
$$

and so, by Schwarz' inequality,

$$
\begin{aligned}
l(r)^{2} & \leqq\left(\int_{0}^{2 \pi} \sqrt{K J_{f}} r d \vartheta\right)^{2} \leqq 2 \pi \int_{0}^{2 \pi} K J_{f} r^{2} d \vartheta \\
& \leqq 2 \pi \int_{0}^{2 \pi}\left[K\left(z_{0}\right)+\omega\left(r ; z_{0}, K\right)\right] J r^{2} d \vartheta \\
& =2 \pi r\left[K\left(z_{0}\right)+\omega\left(r ; z_{0}, K\right)\right] A^{\prime}(r)
\end{aligned}
$$

for a.e. $r$. We use the isoperimetric inequality: $4 \pi A(r) \leqq l(r)^{2}$. Then

$$
\frac{A^{\prime}(r)}{A(r)} \geqq \frac{2}{r\left[K\left(z_{0}\right)+\omega\left(r ; z_{0}, K\right)\right]} \geqq \frac{2}{K\left(z_{0}\right) r}-\frac{2 \omega\left(r ; z_{0}, K\right)}{K\left(z_{0}\right)^{2} r}
$$

and 


$$
\left(\log \left[r^{-2 / K\left(z_{0}\right)} A(r) \exp \left\{2 K\left(z_{0}\right)^{-2} \int_{0}^{r}\left(\omega\left(\varrho ; z_{0}, K\right) / \varrho\right) d \varrho\right\}\right]\right)^{\prime} \geqq 0
$$

for a.e. $r$. Consequently, $B\left(r ; z_{0}, K, f\right)$ is positive and nonincreasing as $r \rightarrow 0$. Therefore, its limit as $r \rightarrow 0$ exists and clearly is the same as $\lim _{r \rightarrow 0} r^{-2 / K\left(z_{0}\right)} A(r)$.

$\mathrm{Re} \mathrm{mark}$. Under the hypotheses of Lemma 1, it follows that the limit in $(2.5)$ exists. Since $f^{-1}$ is $\left(K \circ f^{-1}\right)(w)$-q.c., it will also follow that the (possibly infinite) limit in (2.6) exists, once we verify that (2.4) holds for $\omega\left(r, f\left(z_{0}\right), K \circ f^{-1}\right)$. But this follows from the Hölder continuity of $f^{-1}$ (see $(2.7))$, since

$$
\omega\left(r ; f\left(z_{0}\right), K \circ f^{-1}\right) \leqq \omega\left(a r^{\alpha} ; z_{0}, K\right)
$$

(with $\alpha=1 /\|K\|_{\infty}$ and $a=c_{E}^{-\alpha}$ ).

In order to produce extremal functions for extremum problems that follow, we shall need semicontinuity properties of $\Phi$ and $\Psi$ :

L e m m a 2. Suppose that $K$ satisfies (2.4) at $z_{0} \in D$. Then the functional $\Phi\left(z_{0}, z_{0} ; K, \cdot\right)$ is upper semicontinuous and the functional $\Psi\left(z_{0}, z_{0} ; K, \cdot\right)$ is lower semicontinuous under locally uniform convergence in $Q(K, D)$.

Proof. Suppose that $f_{n}, f \in Q(K, D)$ and $f_{n} \rightarrow f$ uniformly on compact subsets of $D$ as $n \rightarrow \infty$. Let $\varepsilon>0$. Since

$$
\lambda=\lim _{r \rightarrow 0} r^{-2 / K\left(z_{0}\right)} A\left(r ; z_{0}, f\right)=\lim _{r \rightarrow 0} B\left(r ; z_{0}, K, f\right)
$$

exists by Lemma 1, there is an $r_{0}>0$ such that

$$
B\left(r_{0} ; z_{0}, K, f\right)<\lambda+\varepsilon / 2 \text {. }
$$

The q.c. mappings $f_{n}$ carry the circle $\left|z-z_{0}\right|=r_{0}$ onto Jordan curves $C_{n}$, that need not be rectifiable, but have zero area ([2]). Since $f_{n} \rightarrow f$ uniformly on $\left|z-z_{0}\right|=r_{0}$, the areas $A\left(r_{0} ; z_{0}, f_{n}\right)$ converge to $A\left(r_{0} ; z_{0}, f\right)$ as $n \rightarrow \infty$. Therefore

$$
B\left(r_{0} ; z_{0}, K, f_{n}\right)<B\left(r_{0} ; z_{0}, K, f\right)+\varepsilon / 2<\lambda+\varepsilon
$$

for all $n$ sufficiently large. Now the monotonicity of Lemma 1 implies

$$
\lim _{r \rightarrow 0} r^{-2 / K\left(z_{0}\right)} A\left(r ; z_{0}, f_{n}\right)=\lim _{r \rightarrow 0} B\left(r ; z_{0}, K, f_{n}\right)<\lambda+\varepsilon
$$

for all $n$ sufficiently large. This proves the upper semicontinuity of $\Phi\left(z_{0}, z_{0} ; K, \cdot\right)$.

The lower semicontinuity of $\Psi$ will follow from a similar argument in terms of the inverse mappings. For, if $f_{n} \rightarrow f$ locally uniformly, then 
$f_{n}^{-1} \rightarrow f^{-1}$ locally uniformly also. We observed in (3.1) that condition (2.4) implies a similar condition for $\omega\left(r ; f\left(z_{0}\right), K \circ f^{-1}\right)$. In fact, since the Hölder parameters $a$ and $\alpha$ can be chosen uniformly for $f_{n}^{-1}$ at $f_{n}\left(z_{0}\right)$, the integrals

$$
\int_{0}^{r}\left(\omega\left(\varrho ; f_{n}\left(z_{0}\right), K \circ f_{n}^{-1}\right) / \varrho\right) d \varrho
$$

can be estimated uniformly in terms of $\int_{0}^{r}\left(\omega\left(\varrho ; z_{0}, K\right) / \varrho\right)$ d. Since the areas $A\left(r_{0} ; f_{n}\left(z_{0}\right), f_{n}^{-1}\right)$ still converge to $A\left(r_{0} ; f\left(z_{0}\right), f^{-1}\right)$, we obtain

$$
\lim _{r \rightarrow 0} r^{-2 / K\left(z_{0}\right)} A\left(r ; f_{n}\left(z_{0}\right), f_{n}^{-1}\right) \leqq \lim _{r \rightarrow 0} r^{-2 / K\left(z_{0}\right)} A\left(r ; f\left(z_{0}\right), f^{-1}\right)+\varepsilon
$$

for all $n$ sufficiently large, just as in (3.2). The lower semicontinuity of $\Psi\left(z_{0}, z_{0} ; K, \cdot\right)$ follows.

\section{4. $K(z)$-q.c. mappings for which $\Phi\left(z_{0}, z_{0} ; K, f\right)>0$ and $\Psi\left(z_{0}, z_{0} ; K, g\right)<\infty$}

L e m m a 3. Suppose that $K$ satisfies (2.4) at $z_{0} \in D$. Then there exist $f, g \in Q(K, D)$ such that

$$
\Phi\left(z_{0}, z_{0} ; K, f\right)>0 \text { and } \Psi\left(z_{0}, z_{0} ; K, g\right)<\infty .
$$

Proof. Without loss of generality, we may assume that $D=\bar{C}$ and $z_{0}=0$. Let $r_{0}$ be any (small) positive number. We shall construct examples that are conformal for $|z|>r_{0}$.

Define

$$
\hat{\omega}(r)= \begin{cases}\operatorname{ess} \sup \left[K_{0}-K(z)\right] & \text { for } r<r_{0} \\ |z| \leq r & \text { for } r \geqq r_{0} \\ K_{0}-1 & \end{cases}
$$

where $K_{0}=K(0)$. Then $1 \leqq K_{0}-\hat{\omega}(|z|) \leqq K(z)$ for a.e. $z$; and $\int_{0}^{r_{0}}(\hat{\omega}(r) / r) d r<\infty \quad$ since $0 \leqq \hat{\omega}(r) \leqq \omega(r ; 0, K)$ for $r<r_{0}$. Now define

$$
f(z)=\left\{\begin{array}{cl}
z|z|^{\left(1 / K_{0}\right)-1} \exp \left\{\int_{0}^{|r|} \frac{\hat{\omega}(r) d r}{K_{0} r\left[K_{0}-\hat{\omega}(r)\right]}\right\} & \text { for }|z|<r_{0} \\
z r_{0}{ }^{\left(1 / K_{0}\right)-1} \exp \left\{\int_{0}^{r_{0}} \frac{\hat{\omega}(r) d r}{K_{0} r\left[K_{0}-\hat{\omega}(r)\right]}\right\} & \text { for }|z| \geqq r_{0}
\end{array}\right.
$$


and

$$
g(z)= \begin{cases}z|z|^{K_{0}-1} \exp \left\{-\int_{0}^{|z|} \frac{\hat{\omega}(r)}{r} d r\right\} & \text { for }|z|<r_{0} \\ z r_{0}{ }^{K_{0}-1} \exp \left\{-\int_{0}^{r_{0}} \frac{\hat{\omega}(r)}{r} d r\right\} & \text { for }|z| \geqq r_{0} .\end{cases}
$$

Since $|f|$ is increasing as $|z|$ increases and $\arg f=\arg z$, it is evident that $f$ is a homeomorphism of $\bar{C}$ onto $\bar{C}$. Note that

$$
\frac{d}{d r} \log |g|=\frac{K_{0}-\hat{\omega}(r)}{r}>0 .
$$

Therefore $|g|$ also increases as $|z|$ increases and $\arg g=\arg z$. Consequently, $g$ is also a homeomorphism of $\bar{C}$ onto $\bar{C}$. One easily verifies that

$$
\frac{\left|f_{\dot{z}}(z)\right|+\left|f_{\bar{z}}(z)\right|}{\left|f_{z}(z)\right|-\left|f_{\bar{z}}(z)\right|}=\frac{\left|g_{z}(z)\right|+\left|g_{\bar{z}}(z)\right|}{\left|g_{\dot{z}}(z)\right|-\left|g_{\bar{z}}(z)\right|}=K_{0}-\hat{\omega}(|z|) \leqq K(z) \text { a.e. }
$$

Therefore $f$ and $g$ belong to $Q(K)$. It is evident that

$$
\Phi(0,0 ; K, f)=\Psi(0,0 ; K, g)=1 .
$$

By composing finitely many mappings of the form that we have constructed, the following becomes an immediate corollary:

L e m m 4. Suppose that $K$ satisfies (2.4) at $z_{1}, \ldots, z_{N} \in D$. Then there exist $f, g \in Q(K, D)$ such that $\Phi\left(z_{n}, z_{n} ; K, f\right)>0$ and $\Psi\left(z_{n}, z_{n} ; K, g\right)<\infty$ for $n=1, \ldots, N$.

\section{Existence and representation for fundamental solutions}

In this section we assume that $D=\bar{C}$, that $K(z)$ satisfies (2.4) at $z_{0} \in C$, and that $K(1 / z)$ satisfies (2.4) at the origin. Let $Q_{\bar{\Phi}}(K)$ and $Q_{\Psi}(K)$ be those subclasses of $Q(K)$ consisting of functions normalized at $\infty$ by the conditions

$$
\Phi(\infty, \infty ; K, f)=1 \text { and } \Psi(\infty, \infty ; K, f)=1,
$$

respectively. Here we define

$$
\begin{aligned}
& \Phi(\infty, \infty ; K, f)=1 / \Phi(0,0 ; K(1 / z), 1 / f(1 / z)), \\
& \Psi(\infty, \infty ; K, f)=1 / \Psi(0,0 ; K(1 / z), 1 / f(1 / z)) .
\end{aligned}
$$


We shall consider the problems

$$
\max _{Q_{\Phi}(K)} \Phi\left(z_{0}, z_{0} ; K, f\right) \quad \text { and } \min _{Q_{\Psi}(K)} \Psi\left(z_{0}, z_{0} ; K, f\right) .
$$

L e m ma 5. There exist $f \in Q_{\Phi}(K)$ and $g \in Q_{\Psi}(K)$ such that

$$
\begin{aligned}
& 0<\Phi\left(z_{0}, z_{0} ; K, f\right)=\max _{h \in Q_{\Phi}(K)} \Phi\left(z_{0}, z_{0} ; K, h\right) \text { and } \\
& \Psi\left(z_{0}, z_{0} ; K, g\right)=\min _{h \in Q_{\Psi}(K)} \Psi\left(z_{0}, z_{0} ; K, h\right)<\infty .
\end{aligned}
$$

Proof. It follows from Lemma 3 that $Q_{\Phi}(K)$ and $Q_{\Psi}(K)$ are nonempty and that the corresponding supremum and infimum are not 0 and $\infty$, respectively. To see that the maximum and minimum actually exist, let $f_{n} \in Q_{\Phi}(K)$ and $g_{n} \in Q_{\Psi}(K)$ be extremum sequences. Since $\Phi$ and $\Psi$ are invariant under translation, we may assume that $f_{n}\left(z_{1}\right)=g_{n}\left(z_{1}\right)=0$ for a fixed point $z_{1} \neq z_{0}$. Then the $\|K\|_{\infty}$-q.c. families $\left\{f_{n}\right\}$ and $\left\{g_{n}\right\}$ are normal on $C-\left\{z_{1}\right\}$ in the spherical metric ([2]). The normalization at $\infty$ rules out the convergence of any subsequence of $\left\{f_{n}\right\}$ or $\left\{g_{n}\right\}$ to $\infty$ on $C-\left\{z_{1}\right\}$. Similarly, extremality of the sequence at $z_{0}$ rules out the convergence of any subsequence of $\left\{f_{n}\right\}$ or $\left\{g_{n}\right\}$ to a constant on $C-\left\{z_{1}\right\}$. Therefore subsequences of $\left\{f_{n}\right\}$ and $\left\{g_{n}\right\}$ converge locally uniformly on $C$ to $\|K\|_{\infty}$-q.c. mappings $f_{0}$ and $g_{0}$, respectively. It follows from a result of K. Strebel [10] that $f_{0}$ and $g_{0}$ are actually $K(z)$-q.c.; hence $f_{0}, g_{0} \in Q(K)$. The semicontinuity of Lemma 2 implies that

$$
\begin{gathered}
\Phi\left(z_{0}, z_{0} ; K, f_{0}\right) \geqq \sup _{h \in Q_{\Phi}(K)} \Phi\left(z_{0}, z_{0} ; K, h\right), \\
\Psi\left(z_{0}, z_{0} ; K, g_{0}\right) \leqq \inf _{h \in Q_{\Psi^{\prime}}(K)} \Psi\left(z_{0}, z_{0} ; K, h\right) \\
0<\Phi\left(\infty, \infty ; K, f_{0}\right) \leqq 1, \quad 1 \leqq \Psi\left(\infty, \infty ; K, g_{0}\right)<\infty .
\end{gathered}
$$

Since $f_{0} / \Phi\left(z_{0}, z_{0} ; K, f_{0}\right) \in Q_{\Phi}(K)$ could only increase the maximum and $g_{0} / \Psi\left(z_{0}, z_{0} ; K, g_{0}\right) \in Q_{\Psi}(K)$ could only decrease the minimum, necessarily $f_{0} \in Q_{\Phi}(K)$ and attains the maximum, and $g_{0} \in Q_{\Psi}(K)$ and attains the minimum.

The or em 1. Suppose that $K: \bar{C} \rightarrow[1, \infty)$ is measurable and $\|K\|_{\infty}<\infty$. Assume that $K(z)$ satisfies (2.4) at $z_{0} \in C$ and $K(1 / z)$ satisfies (2.4) at the origin. Then there exists a $K(z)$-q.c. mapping $f$ such that

$$
S\left(z, z_{0}\right)=-\log \left|f(z)-f\left(z_{0}\right)\right|
$$

is a (weak) fundamental solution of the differential equation

$$
\operatorname{div}(K \operatorname{grad} U)=0
$$

with singularities at $z_{0}$ and $\infty$. Moreover, 


$$
s\left(z, z_{0}\right)=S\left(z, z_{0}\right)+\frac{1}{K\left(z_{0}\right)} \log \left|z-z_{0}\right|
$$

has a limit as $z \rightarrow z_{0}$, and

$$
t\left(z, z_{0}\right)=S\left(z, z_{0}\right)+\frac{1}{K(\infty)} \log |z|
$$

has a limit as $z \rightarrow \infty$. Similarly, there exists a $K(z)$-q.c. mapping $g$ such that

$$
\Sigma\left(z, z_{0}\right)=-\log \left|g(z)-g\left(z_{0}\right)\right|
$$

is a (weak) fundamental solution of the differential equation

$$
\operatorname{div}((1 / K) \operatorname{grad} U)=0
$$

with singularities at $z_{0}$ and $\infty$. Moreover,

$$
\sigma\left(z, z_{0}\right)=\Sigma\left(z, z_{0}\right)+K\left(z_{0}\right) \log \left|z-z_{0}\right|
$$

has a limit as $z \rightarrow z_{0}$, and

$$
\tau\left(z, z_{0}\right)=\Sigma\left(z, z_{0}\right)+K(\infty) \log |z|
$$

has a limit as $z \rightarrow \infty$.

$\mathrm{R}$ e $\mathrm{m}$ a r ks. The principal impact of Theorem 1 is that the differential equations (5.2) and (5.6) have fundamental solutions representable in terms of quasiconformal mappings. That is,

$$
f=f\left(z_{0}\right)+e^{-[S+i T]}
$$

where

$$
K \frac{\partial S}{\partial x}=\frac{\partial T}{\partial y}, \quad K \frac{\partial S}{\partial y}=-\frac{\partial T}{\partial x}
$$

and

$$
g=g\left(z_{0}\right)+e^{-[\Sigma+i x]}
$$

where

$$
\frac{\partial \Sigma}{\partial x}=K \frac{\partial r}{\partial y}, \quad \frac{\partial \Sigma}{\partial y}=-K \frac{\partial r}{\partial x} .
$$

However, the $K(z)$-q.c. mappings $f$ and $g$ may change as $z_{0}$ varies.

If we view the plane as a nonhomogeneous dielectric medium with dielectric coefficient $K=K(z) \geqq 1$, then $S\left(z, z_{0}\right)$ represents the electrostatic potential at $z$ of a point charge at $z_{0}$. It is called the dielectric Green's function. (If the dielectric coefficient is between 0 and 1 , it can 
be represented by $1 / K(z)$, where $K(z) \geqq 1$; in this case $\Sigma\left(z, z_{0}\right)$ is the dielectric Green's function.)

For example, suppose $C=\cup_{n=0}^{N} \overline{D_{n}}$ where the $D_{n}$ are disjoint domains, which we think of as isotropic dielectric media with dielectric constants $K_{n} \geqq 1$. Assume that $D_{0}$ contains a neighborhood of $\infty$. If $z_{0} \in D_{v}$, then $S\left(z, z_{0}\right)$ is a harmonic function of $z$ in each $D_{n}$, except at $z_{0}$, where $S\left(z, z_{0}\right)+\left(1 / K_{v}\right) \log \left|z-z_{0}\right|$ is continuous. Moreover, $S\left(z, z_{0}\right)$ is continuous in $C$ and $S\left(z, z_{0}\right)+\left(1 / K_{0}\right) \log |z| \rightarrow 0$ as $z \rightarrow \infty$. Furthermore, if $D_{m}$ and $D_{n}$ have a smooth common boundary arc $\gamma$ with normal $n_{z}$ at $z \in \gamma$, then

$$
K_{m} \frac{\partial}{\partial n_{z}}\left[\left.S\left(z, z_{0}\right)\right|_{D_{m}}\right]=K_{n} \frac{\partial}{\partial n_{z}}\left[\left.S\left(z, z_{0}\right)\right|_{D_{n}}\right] .
$$

The case $K_{0}=1, K_{1}=K_{2}=\ldots=K_{N}$, was treated in [5]. In this special case a univalent representation for $S$ and $\Sigma$ was obtained by other means.

Proof of Theorem 1. Let $f$ be the function from Lemma 5 that maximizes the functional $\Phi\left(z_{0}, z_{0} ; K, \cdot\right)$ over $Q_{\Phi}(K)$. Consider q.c. variations of $f$, as in [9, Chapter 13], of the form

$$
w^{*}=w-\frac{\varepsilon}{\pi} \iint_{C} \frac{a(\omega)}{\omega-w} d m_{\omega}+O\left(\varepsilon^{2}\right),
$$

where $a$ has compact support in $C-f\left(\left|z-z_{0}\right| \leqq r\right)$ and $m$ is Lebesgue measure. Under this variation the normalization at $\infty$ is preserved and the area

$$
\begin{aligned}
& A\left(r ; z_{0}, f^{*}\right) \\
& =A\left(r ; z_{0}, f\right)-2 \operatorname{Re} \frac{\varepsilon}{\pi} \int_{f\left(\left|z-z_{0}\right|<r\right)} \iint_{C} \frac{a(\omega)}{(\omega-w)^{2}} d m_{\omega} d m_{w}+O\left(\varepsilon^{2}\right) .
\end{aligned}
$$

It follows that

$$
\begin{aligned}
& \Phi\left(z_{0}, z_{0} ; K, f^{*}\right) \\
& =\Phi\left(z_{0}, z_{0} ; K, f\right)-\varepsilon \Phi\left(z_{0}, z_{0} ; K, f\right) \operatorname{Re} \frac{1}{\pi} \iint_{C} \frac{a(\omega) d m_{\omega}}{\left[\omega-f\left(z_{0}\right)\right]^{2}}+O\left(\varepsilon^{2}\right)
\end{aligned}
$$

under variations of the form (5.13). Then the principal theorem of the variational method [9, Theorem 13.2] implies that $f$ satisfies the differential equation

$$
f_{\bar{z}}(z)=\frac{K(z)-1}{K(z)+1} \cdot \frac{-\left[f(z)-f\left(z_{0}\right)\right]^{2}}{\left|f(z)-f\left(z_{0}\right)\right|^{2}} \overline{f_{z}(z)} \text { a.e. }
$$

That is, if 


$$
\log \frac{1}{f-f\left(z_{0}\right)}=S+i T
$$

then

$$
(S+i T)_{\bar{z}}=-\frac{K-1}{K+1} \overline{(S+i T)_{z}} \text { a.e. }
$$

Consequently, $S$ and $T$ satisfy the generalized Cauchy-Riemann equations (5.10), and $S$ satisfies the differential equation (5.2).

A similar argument with the function $g$ from Lemma 5 leads to the differential equation

$$
(\Sigma+i \Upsilon)_{\bar{z}}=\frac{K-1}{K+1} \overline{(\Sigma+i \Upsilon)_{z}}
$$

for

$$
\Sigma+i r=\log \frac{1}{g-g\left(z_{0}\right)} .
$$

Thus $\Sigma$ and $\Upsilon$ satisfy the generalized Cauchy-Riemann equations (5.12), and $\Sigma$ satisfies (5.6).

It is evident from (5.1) and (5.5) that $S$ and $\Sigma$ have singularities at $z_{0}$ and $\infty$. To see that they are of the form indicated in $(5.3)-(5.4)$ and (5.7)-(5.8), let us consider first the singularity of $S$ at $z_{0}$. Let $\Delta$ be a disk of radius $\delta$ with center $z_{0}$. Assume $z \in \Delta$ and let $\Delta_{\varrho}$ be obtained from $\Delta$ by deleting disks of radius $\varrho$ about $z_{0}$ and $z$. First use (5.10) and Green's theorem (see, e.g., [2, p. 148]) to evaluate

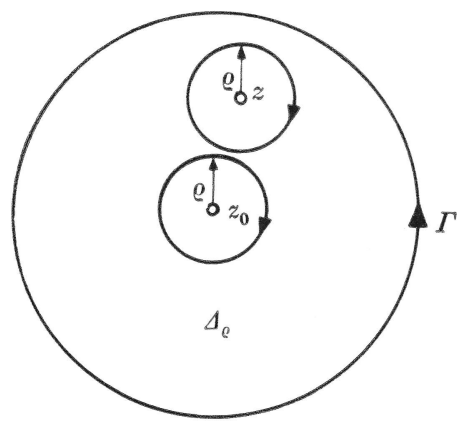

$$
\begin{gathered}
\iint_{\Delta_{\varrho}} K(\zeta) \operatorname{grad}_{\zeta} S\left(\zeta, z_{0}\right) \cdot \operatorname{grad}_{\zeta} \log |z-\zeta| d m_{\zeta}=\int_{\partial \Delta_{\varrho}} \log |z-\zeta| d T\left(\zeta, z_{0}\right) \\
\rightarrow 2 \pi \log \left|z-z_{0}\right|+\int_{\Gamma^{\prime}} \log |z-\zeta| d T\left(\zeta, z_{0}\right) \quad \text { as } \varrho \rightarrow 0 .
\end{gathered}
$$

Similarly, 


$$
\begin{aligned}
& \iint_{\Delta_{Q}} K\left(z_{0}\right) \operatorname{grad}_{\zeta} S\left(\zeta, z_{0}\right) \cdot \operatorname{grad}_{\zeta} \log |\zeta-z| d m_{\zeta} \\
& =K\left(z_{0}\right) \int_{\partial \Delta_{\varrho}} S\left(\zeta, z_{0}\right) d \arg (\zeta-z) \\
& \rightarrow-2 \pi K\left(z_{0}\right) S\left(z, z_{0}\right)+K\left(z_{0}\right) \int_{\Gamma} S\left(\zeta, z_{0}\right) d \arg (\zeta-z) \text { as } \varrho \rightarrow 0 .
\end{aligned}
$$

Therefore

$$
\begin{array}{r}
s\left(z, z_{0}\right)=\frac{1}{2 \pi} \int_{\Gamma} S\left(\zeta, z_{0}\right) d \arg (\zeta-z)-\frac{1}{2 \pi K\left(z_{0}\right)} \int_{\Gamma} \log |z-\zeta| d T\left(\zeta, z_{0}\right) \\
+\frac{1}{2 \pi K\left(z_{0}\right)} I(z)
\end{array}
$$

where

$$
I(z)=\iint_{\Delta}\left[K(\zeta)-K\left(z_{0}\right)\right] \operatorname{grad}_{\zeta} S\left(\zeta, z_{0}\right) \cdot \operatorname{grad}_{\zeta} \log |\zeta-z| d m_{\zeta} .
$$

Evidently, $s\left(\cdot, z_{0}\right)$ is continuous at $z_{0}$ if $I$ is. First, $I\left(z_{0}\right)$ converges since

$$
\begin{aligned}
\left|I\left(z_{0}\right)\right|^{2} & \leqq\left[\iint_{\Delta} \frac{\omega\left(\left|\zeta-z_{0}\right|\right)}{\left|\zeta-z_{0}\right|} \frac{|| d f(\zeta)|| d m_{\zeta}}{\left|f(\zeta)-f\left(z_{0}\right)\right|}\right]^{2} \\
& \leqq \frac{2 \pi}{\alpha}\left(\int_{0}^{\delta} \frac{\omega(r)}{r} d r\right)\left(\iint \frac{\omega(r) J_{f}(\zeta) d m_{\zeta}}{\left|f(\zeta)-f\left(z_{0}\right)\right|^{2}}\right) \\
& \leqq \frac{4 \pi^{2}}{\alpha} \int_{0}^{\delta} \frac{\omega(r)}{r} d r \int_{0}^{\delta^{\prime}} \frac{\omega\left(a R^{\alpha}\right)}{R} d R=\frac{4 \pi^{2}}{\alpha^{2}} \int_{0}^{\delta} \frac{\omega(r)}{r} d r \int_{0}^{\delta^{\prime \prime}} \frac{\omega(\varrho)}{\varrho} d \varrho<\infty
\end{aligned}
$$

where $\alpha=1 /\|K\|_{\infty}$. Finally, we estimate

$$
\begin{aligned}
\mid I(z)- & \left.I\left(z_{0}\right)\right|^{2} \leqq\left[\left|z-z_{0}\right| \iint_{\Delta} \frac{\omega\left(\left|\zeta-z_{0}\right|\right)|| d f(\zeta)||}{\left|\zeta-z_{0}\right||\zeta-z|\left|f(\zeta)-f\left(z_{0}\right)\right|} d m_{\zeta}\right]^{2} \\
& \leqq \\
& \left|z-z_{0}\right|^{2} \iint \frac{\omega\left(\left|\zeta-z_{0}\right|\right) d m_{\zeta}}{\left|\zeta-z_{0}\right|^{2}|\zeta-z|^{2-\alpha}} \int \frac{\omega\left(\left|\zeta-z_{0}\right|\right)|| d f(\zeta)||^{2} d m_{\zeta}}{|\zeta-z|^{\alpha}\left|f(\zeta)-f\left(z_{0}\right)\right|^{2}} \\
& \leqq \\
& \int_{0}^{\delta} \int_{0}^{\delta} \frac{\omega(r) d \vartheta d r}{r\left|r e^{i \theta}\left(z-z_{0}\right)^{-1}-1\right|^{2-\alpha}} \int_{0}^{\delta^{\prime}} \int_{0}^{2 \pi} \frac{\omega\left(a R^{\alpha}\right) d \Theta d R}{R\left|\left(\zeta-z_{0}\right)\left(z-z_{0}\right)^{-1}-1\right|^{\alpha}}
\end{aligned}
$$




$$
\begin{aligned}
& \leqq(\text { constant }) \text {. } \\
& \int_{0}^{\delta} \int_{0}^{2 \pi} \frac{\omega(r) d \vartheta d r}{r\left|r e^{i \eta \vartheta}\left(z-z_{0}\right)^{-1}-1\right|^{2-\alpha}} \int_{0}^{\delta^{\prime}} \int_{0}^{2 \pi} \frac{\omega\left(a R^{\alpha}\right) d \Theta d R}{R\left|R e^{i \Theta}\left[f(z)-f\left(z_{0}\right)\right]^{-1}-1\right|} \\
& \leqq \text { (constant }) \text {. } \\
& \int_{0}^{\delta} \frac{\omega(r) d r}{r\left|r^{2}\right| z-\left.z_{0}\right|^{-2}-\left.1\right|^{1-\alpha / 2}} \int_{0}^{\delta^{\prime}} \frac{\omega\left(a R^{\alpha}\right) d R}{R\left|R^{2}\right| f(z)-\left.f\left(z_{0}\right)\right|^{-2}-\left.1\right|^{1 / 2}} \\
& \leqq \text { (constant) } \int_{0}^{\infty} \frac{\omega\left(\left|z-z_{0}\right| t\right) d t}{t\left|t^{2}-1\right|^{1-\alpha / 2}} \int_{0}^{\infty} \frac{\omega\left(\alpha\left|f(z)-f\left(z_{0}\right)\right|^{\alpha} t\right) d t}{t\left|t^{2 / \alpha}-1\right|^{1 / 2}} .
\end{aligned}
$$

Since $\omega(r) \rightarrow 0$ as $r \rightarrow 0$, both of these integrals tend to 0 as $z \rightarrow z_{0}$.

The continuity of $t$ at $\infty$, of $\sigma$ at $z_{0}$, and of $\tau$ at $\infty$ follow from similar considerations.

\section{A distortion theorem for $K(z)$-q.c. mappings of $C$}

In this section we fix $N$ points $z_{1}, \ldots, z_{N} \in D=C$, at which we assume that $K$ satisfies (2.4). We shall measure the mutual distortion relative to these points of a $K(z)$-q.c. mapping $f$ by the quadratic expressions

$$
\begin{aligned}
& \phi[f]=\sum_{m, n=1}^{N} x_{m} x_{n} \log \Phi\left(z_{m}, z_{n} ; K, f\right) \text { and } \\
& \psi[f]=\sum_{m, n=1}^{N} x_{m} x_{n} \log \Psi\left(z_{m}, z_{n} ; K, f\right)
\end{aligned}
$$

where $x_{1}, \ldots, x_{N} \in R$ and $\sum_{n=1}^{N} x_{n}=0$. By solving the problems

$$
\max _{Q(K)} \phi \quad \text { and } \quad \min _{Q(K)} \psi
$$

we shall obtain the following:

Theorem 2. Suppose that $K: C \rightarrow[1, \infty)$ is measurable and $\|K\|_{\infty}<\infty$. Assume that $K(z)$ satisfies (2.4) at $z_{1}, \ldots, z_{n} \in C$ and $K(1 / z)$ satisfies (2.4) at the origin. If $f: C \rightarrow C$ is a $K(z)$-q.c. mapping, then

$$
\sum_{m, n=1}^{N} x_{m} x_{n}\left[\log \frac{\left|f\left(z_{m}\right)-f\left(z_{n}\right)\right|}{\left|z_{m}-z_{n}\right|^{\left[1 / K\left(z_{m}\right)+1 / K\left(z_{n}\right)\right] / 2}}+s\left(z_{m}, z_{n}\right)\right] \leqq 0
$$

and 


$$
\sum_{m, n=1}^{N} x_{m} x_{n}\left[\log \frac{\left|f\left(z_{m}\right)-f\left(z_{n}\right)\right|}{\left|z_{m}-z_{n}\right|^{\left[K\left(z_{m}\right)+K\left(z_{n}\right)\right] / 2}}+\sigma\left(z_{m}, z_{n}\right)\right] \geqq 0
$$

for any $x_{1}, \ldots, x_{N} \in \boldsymbol{R}$ subject to the constraint $\sum_{n=1}^{N} x_{n}=0$. Here $s$ and $\sigma$ are the reqular parts (5.3) and (5.7) of fundamental solutions to (5.2) and (5.6), respectively.

Of course, we interpret the quotients in (6.3) and (6.4) for $m=n$ as $\Phi\left(z_{n}, z_{n} ; K, f\right)$ and $\Psi\left(z_{n}, z_{n} ; K, f\right)$, respectively.

Proof of Theorem 2. It follows from Lemma 4 that $-\infty<\sup _{Q(K)} \phi$ and $\inf _{Q(K)} \psi<+\infty$. For $m \neq n$ it is obvious that $\Phi\left(z_{m}, z_{n} ; K, \cdot\right)$ and $\Psi\left(z_{m}, z_{n} ; K, \cdot\right)$ are continuous under locally uniform convergence. Together with Lemma 2, this implies that $\phi$ and $\psi$ are upper and lower semicontinuous, respectively, on $Q(K)$. Since the functionals $\phi$ and $\psi$ are invariant when replacing $f$ by $A f+B$, it is sufficient to restrict attention to the subset of $Q(K)$ for which $f(0)=0$ and $f(1)=1$. By a theorem of K. Strebel [10], this subset is compact in the topology of locally uniform convergence. Therefore $f, g \in Q(K)$ exist such that

$$
\phi[f]=\max _{Q(K)} \phi \quad \text { and } \quad \psi[g]=\min _{Q(K)} \psi .
$$

We shall again employ variations of the form (5.13). Under this variation $\log \Phi\left(z_{m}, z_{n} ; K, f^{*}\right)$

$$
=\log \Phi\left(z_{m}, z_{n} ; K, f\right)-\varepsilon \operatorname{Re}\left\{\frac{1}{\pi} \iint \frac{a(\omega) d m_{\omega}}{\left[\omega-f\left(z_{m}\right)\right]\left[\omega-f\left(z_{n}\right)\right]}\right\}+O\left(\varepsilon^{2}\right)
$$

and

$$
\phi\left[f^{*}\right]=\phi[f]-\varepsilon \operatorname{Re}\left\{\frac{1}{\pi} \iint_{C} a(\omega)\left[\sum_{n=1}^{N} \frac{x_{n}}{\omega-f\left(z_{n}\right)}\right]^{2} d m_{\omega}\right\}+O\left(\varepsilon^{2}\right) .
$$

It follows then from the principal theorem on the variational method [9, Theorem 13.2] that $f$ satisfies the differential equation

$$
f_{\bar{z}}(z)=\frac{K(z)-1}{K(z)+1} \cdot \frac{\left|\sum_{n=1}^{N} x_{n} /\left[f(z)-f\left(z_{n}\right)\right]\right|^{2}}{-\left[\sum_{n=1}^{N} x_{n} /\left[f(z)-f\left(z_{n}\right)\right]\right]^{2}} \overline{f_{z}(z)} .
$$

That is, if

$$
\sum_{n=1}^{N} x_{n} \log \frac{1}{f-f\left(z_{n}\right)}=F=U+i V
$$

then 


$$
F_{\bar{z}}=-\frac{K-1}{K+1} \overline{F_{z}}
$$

An argument similar to that in the proof of Theorem 1 implies that the singularities of $U$ are such that

$$
U(z)+\frac{x_{n}}{K\left(z_{n}\right)} \log \left|z-z_{n}\right|
$$

has a limit as $z \rightarrow z_{n}$. Consequently,

$$
W(z)=F^{\prime}(z)-\sum_{n=1}^{N} x_{n}\left[S\left(z, z_{n}\right)+i T\left(z, z_{n}\right)\right]
$$

is continuous in the whole plane and satisfies

$$
W_{\bar{z}}=-\frac{K-1}{K+1} \overline{W_{z}} \text { a.e. }
$$

In addition, (5.4) and the fact that $\sum_{n=1}^{N} x_{n}=0$ imply that $W$ has a finite limit at $\infty$, too. If $W=u+i v$ and $\Delta_{R}=\left\{z: 1 / R<\left|z-z_{m}\right|<R\right\}$, then on the one hand,

$$
\int_{\Delta_{R}} \int K(z) \operatorname{grad} S\left(z, z_{m}\right) \cdot \operatorname{grad} u(z) d m_{z}=\int_{\partial \Delta_{R}} S\left(z, z_{m}\right) d v(z) \rightarrow 0 \quad \text { as } R \rightarrow \infty
$$

and, on the other hand,

$$
\begin{aligned}
& \int_{\Delta_{R}} \int_{\Delta_{R}} K(z) \operatorname{grad} S\left(z, z_{m}\right) \cdot \operatorname{grad} u(z) d m_{z} \\
& =\int_{\partial \Delta_{R}} u(z) d T\left(z, z_{m}\right) \rightarrow 2 \pi\left[u\left(z_{m}\right)-u(\infty)\right]
\end{aligned}
$$

as $R \rightarrow \infty$. That is, $\sum_{m=1}^{N} x_{m} u\left(z_{m}\right)=u(\infty) \sum_{m=1}^{N} x_{m}=0$. Consequently,

$$
\begin{gathered}
\sum_{m, n=1}^{N} x_{m} x_{n} \log \frac{\left|f\left(z_{m}\right)-f\left(z_{n}\right)\right|}{\left|z_{m}-z_{n}\right| 1 / K\left(z_{n}\right)}=\sum_{m=1}^{N} x_{m}\left\{-U\left(z_{m}\right)+\sum_{n=1}^{N} x_{n}\left[S\left(z_{m}, z_{n}\right)-s\left(z_{m}, z_{n}\right)\right]\right\} \\
=-\sum_{m=1}^{N} x_{m} u\left(z_{m}\right)-\sum_{m, n=1}^{N} x_{m} x_{n} s\left(z_{m}, z_{n}\right)=-\sum_{m, n=1}^{N} x_{m} x_{n} s\left(z_{m}, z_{n}\right)
\end{gathered}
$$

and

$$
\phi[f]=-\frac{1}{2} \sum_{m, n=1}^{N} x_{m} x_{n}\left[s\left(z_{m}, z_{n}\right)+s\left(z_{n}, z_{m}\right)\right]=-\sum_{m, n=1}^{N} x_{m} x_{n} s\left(z_{m}, z_{n}\right)
$$

in the extreme case. This completes the proof of (6.3). 
The proof of (6.4) is quite similar. The variational formula for $\psi$ is the same as for $\phi$ in (6.5). However, since we must maximize $-\psi$, the sign in (6.6) and (6.8) changes. This has the effect of replacing $K$ by $1 / K$, and hence, $s$ by $\sigma$ in the final result.

R e mark. The proof of Theorem 2 provides both existence and a representation in terms of q.c. mappings for solutions of equations (5.2) and (5.6) with singularities at finitely many points. For example, with $x_{1}=-1, x_{2}=1$, and $N=2$, we obtain $K(z)$-q.c. mappings $f$ and $g$ such that

$$
\log \left|\frac{f(z)-f\left(z_{1}\right)}{f(z)-f\left(z_{2}\right)}\right|
$$

is a fundamental solution of (5.2) with singularities

$$
-\frac{1}{K\left(z_{1}\right)} \log \frac{1}{\left|z-z_{1}\right|} \quad \text { and } \quad \frac{1}{K\left(z_{2}\right)} \log \frac{1}{\left|z-z_{2}\right|} \text {, }
$$

and

$$
\log \left|\frac{g(z)-g\left(z_{1}\right)}{g(z)-g\left(z_{2}\right)}\right|
$$

is a fundamental solution of (5.6) with singularities

$$
-K\left(z_{1}\right) \log \frac{1}{\left|z-z_{1}\right|} \text { and } K\left(z_{2}\right) \log \frac{1}{\left|z-z_{2}\right|} .
$$

The following is an application of Theorem 2 :

Corollary 1. Suppose $f: C \rightarrow C$ is a homeomorphism that is $K$-q.c. for $|z|<R$ and $L$-q.c. for $|z|>R$ ( $K, L$ constants). For $m=$ $1, \ldots, M$ and $n=1, \ldots, N$, let $z_{m}$ and $\zeta_{n}$ be any complex numbers with $\left|z_{m}\right|<R$ and $\left|\zeta_{n}\right|>R$, and let $x_{m}$ and $y_{n}$ be any real numbers with $\sum_{m=1}^{M} x_{m}+\sum_{n=1}^{N} y_{n}=0$. Then

$$
\begin{gathered}
\sum_{\mu, m=1}^{M} x_{\mu} x_{m} \log \left[\frac{\left|f\left(z_{\mu}\right)-f\left(z_{m}\right)\right|}{\left|z_{\mu}-z_{m}\right|^{1 / K}} R^{1 / K}\left|1-\frac{z_{\mu} \overline{z_{m}}}{R^{2}}\right|^{(L-K) /[K(L+K)]}\right] \\
+2 \sum_{m=1}^{M} \sum_{n=1}^{N} x_{m} y_{n} \log \left[\frac{\left|f\left(z_{m}\right)-f\left(\zeta_{n}\right)\right|}{\left.\left|z_{m}-\zeta_{n}\right|^{2 /(K+L)} R^{2 /(K+L)}\right]}\right. \\
+\sum_{v, n=1}^{N} y_{\nu} y_{n} \log \left[\frac{\left[f\left(\zeta_{\nu}\right)-f\left(\zeta_{n}\right) \mid\right.}{\left|\zeta_{\nu}-\zeta_{n}\right|^{1 / L}} R^{1 / L}\left|1-\frac{\zeta_{\nu} \bar{\zeta}_{n}}{R^{2}}\right|^{(K-L) /[L(K+L)]}\right] \\
\leqq 0 \leqq
\end{gathered}
$$




$$
\begin{aligned}
& \sum_{\mu, m=1}^{M} x_{\mu} x_{m} \log \left[\frac{\left|f\left(z_{\mu}\right)-f\left(z_{m}\right)\right|}{\left|z_{\mu}-z_{m}\right|^{K}} R^{K}\left|1-\frac{z_{\mu} \overline{z_{m}}}{R^{2}}\right|^{[K(K-L)] /(K+L)}\right] \\
& +2 \sum_{m=1}^{M} \sum_{n=1}^{N} x_{m} y_{n} \log \left[\frac{\left|f\left(z_{m}\right)-f\left(\zeta_{n}\right)\right|}{\left|z_{m}-\zeta_{n}\right|^{2 K L /(K+L)}} R^{2 K L /(K+L)}\right] \\
& +\sum_{v, n=1}^{N} y_{v} y_{n} \log \left[\frac{\left|f\left(\zeta_{\nu}\right)-f\left(\zeta_{n}\right)\right|}{\left|\zeta_{v}-\zeta_{n}\right|^{L}} R^{L}\left|1-\frac{\zeta_{\nu} \bar{\zeta}_{n}}{R^{2}}\right|^{[L(L-K)] /(L+K)}\right] .
\end{aligned}
$$

Proof. For the first inequality, we need fundamental solutions $S$ of (5.2) with singularity $-(1 / L) \log |z|$ at $\infty$ and with singularity $(1 / K) \log \left(1 /\left|z-z_{m}\right|\right)$ at $z_{m}$ or $(1 / L) \log \left(1 /\left|z-\zeta_{n}\right|\right)$ at $\zeta_{n}$. Since $K$ and $L$ are constants, $S$ is to be otherwise harmonic, except for $|z|=R$, where it is continuous. Since $K(z) \partial S / \partial r=(1 / r)(\partial T / \partial \vartheta)$ has no discontinuity on $|z|=R$, we must also have

$$
\left.K \frac{\partial S}{\partial r}\right|_{r=R^{-}}=\left.L \frac{\partial S}{\partial r}\right|_{r=R^{+}} \cdot
$$

From these conditions, one verifies directly that

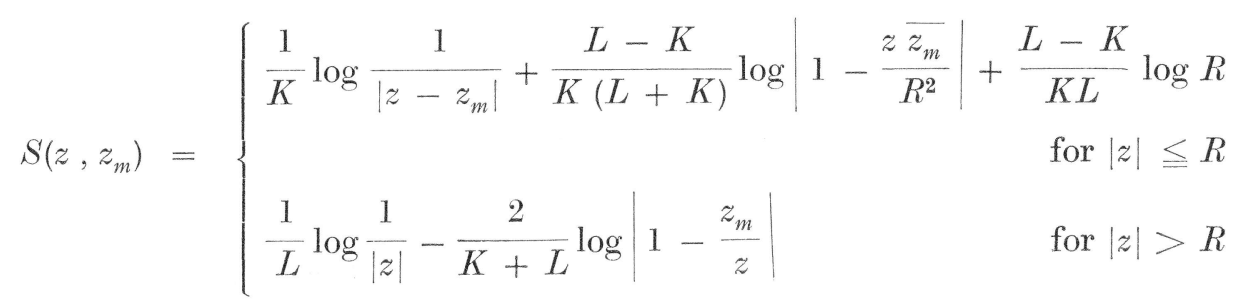

and

$$
S\left(z, \zeta_{n}\right)=\frac{1}{L} \log \frac{1}{\left|z-\zeta_{n}\right|}+\frac{K-L}{L(K+L)} \begin{cases}\log \left|1-\frac{z}{\zeta_{n}}\right| & \text { for }|z| \leqq R \\ \log \left|1-\frac{R^{2}}{z \bar{\zeta}}\right| & \text { for }|z|>R\end{cases}
$$

are fundamental solutions. Substitution of the corresponding expressions

$$
\begin{gathered}
s\left(z_{\mu}, z_{m}\right)=\frac{L-K}{K(L+K)} \log \left|1-\frac{z_{\mu} \overline{z_{m}}}{R^{2}}\right|+\frac{L-K}{K L} \log R, \\
s\left(\zeta_{\nu}, \zeta_{n}\right)=\frac{K-L}{L(K+L)} \log \left|1-\frac{R^{2}}{\zeta_{\nu} \overline{\zeta_{n}}}\right| \\
s\left(z_{m}, \zeta_{n}\right)+s\left(\zeta_{n}, z_{m}\right)=\frac{(K-L)^{2}}{K L(K+L)} \log \left|z_{m}-\zeta_{n}\right|+\frac{2(L-K)}{L(L+K)} \log \left|\zeta_{n}\right|
\end{gathered}
$$

into (6.3) leads to the first inequality. 
The second inequality follows from (6.4) in an entirely analogous fashion, with $K$ and $L$ replaced by their reciprocals.

$\mathrm{R}$ e m a r k s. A special case of Corollary 1 appears in our earlier work [8]. We leave it to the reader to test the special cases when $K=L$ or when all $y_{n}=0$ and $L \rightarrow \infty$ (cf. [8]).

The inequalities of Theorem 2 and Corollary 1 are, of course, sharp. They are a significant generalization of the classical Golusin inequalities, which are a valuable tool in studying conformal mappings.

It is an interesting exercise to construct the $K(z)$-q.c. mappings $f$ and $g$ from Theorem 1 that generate the fundamental solutions $S$ and $\Sigma$, when $K(z) \equiv K$ for $|z|<R$ and $K(z) \equiv L$ for $|z|>R$.

\section{Fundamental solutions of the first kind}

Let $D$ be a domain that admits a harmonic Green's function. Assume that $K: D \rightarrow[1, \infty)$ satisfies $(2.4)$ at a fixed finite point $z_{0} \in D$. As in Section 5 , let $Q_{\Phi}(K, D)$ and $Q_{\Psi}(K, D)$ consist of all functions $f \in Q(K, D)$ that are normalized by $\Phi\left(z_{0}, z_{0} ; K, f\right)=1$ and $\Psi\left(z_{0}, z_{0} ; K, f\right)=1$, respectively.

If $f \in Q(K, D)$, then $\Omega=f(D)$ also admits a harmonic Green's function $G_{f}$. That is, as a function of $w$ :

(1) $G_{f}(w, \zeta)$ is harmonic in $\Omega-\{\zeta\}$,

(2) $\Gamma_{f}(w, \zeta)=G_{f}(w, \zeta)+\log |w-\zeta|$ is harmonic at $\zeta$, and

(3) $G_{f}(w, \zeta) \rightarrow 0$ as $w \rightarrow \partial \Omega$.

In addition $G_{f}$ is symmetric: $G_{f}(w, \zeta)=G_{f}(\zeta, w)$.

Let $\gamma_{f}(w)=\Gamma_{f}(w, w)$. By considering the problems

$$
\min _{Q_{\Phi}(K, D)} \gamma_{f}\left(f\left(z_{0}\right)\right) \quad \text { and } \max _{Q_{\Psi}(K, D)} \gamma_{f}\left(f\left(z_{0}\right)\right)
$$

we shall obtain the following representation theorem:

The orem 3. Let $D$ be a domain that admits a harmonic Green's function. Suppose that $K: D \rightarrow[1, \infty)$ is measurable, is essentially bounded, and satisfies (2.4) at $z_{0} \in D$. Then there exists a $K(z)$-q.c. mapping $f$ of $D$ such that

$$
S\left(z, z_{0} ; D\right)=G_{f}\left(f(z), f\left(z_{0}\right)\right),
$$

where $G_{f}$ is the harmonic Green's function of $f(D)$, is a (weak) fundamental solution of the differential equation

$$
\operatorname{div}(K \operatorname{grad} U)=0
$$

in $D$, with singularity at $z_{0}$ and zero boundary values. Furthermore, 


$$
s\left(z, z_{0} ; D\right)=G_{f}\left(f(z), f\left(z_{0}\right)\right)+\frac{1}{K\left(z_{0}\right)} \log \left|z-z_{0}\right|
$$

has a limit as $z \rightarrow z_{0}$. Similarly, there exists a $K(z)$-q.c. mapping $g$ of $D$ such that

$$
\Sigma\left(z, z_{0} ; D\right)=G_{g}\left(g(z), g\left(z_{0}\right)\right),
$$

where $G_{g}$ is the harmonic Green's function of $g(D)$, is a (weak) fundamental solution of the differential equation

$$
\operatorname{div}((1 / K) \operatorname{grad} U)=0
$$

in D, with singularity at $z_{0}$ and zero boundary values. Furthermore,

$$
\sigma\left(z, z_{0} ; D\right)=G_{g}\left(g(z), g\left(z_{0}\right)\right)+K\left(z_{0}\right) \log \left|z-z_{0}\right|
$$

has a limit as $z \rightarrow z_{0}$.

Proof. Existence of extremal functions $f$ and $g$ for the problems (7.1) presents no difficulty (cf. Lemma 5). We normalize the variations (5.13) so that the points $\infty, f\left(z_{0}\right)$, and the normalization at $f\left(z_{0}\right)$ are preserved. That is, if $w_{0}=f\left(z_{0}\right)$, then

$$
w^{* *}(w)=\left[w^{*}(w)-w^{*}\left(w_{0}\right)\right] / w^{* \prime}\left(w_{0}\right)+w_{0}
$$

is the desired variation, where $w^{*}$ is given in (5.13). Under this variation

$$
\begin{aligned}
& G_{f^{* *}}\left(w^{* *}, w_{0}^{* *}\right)=G_{f^{*}}\left(w^{*}, w_{0}^{*}\right) \\
& =G_{f}\left(w, w_{0}\right)+\operatorname{Re}\left\{\frac{\varepsilon}{\pi} \iint a(\omega) P_{f}^{\prime}(\omega ; w) P_{f}^{\prime}\left(\omega ; w_{0}\right) d m_{\omega}\right\}+o(\varepsilon)
\end{aligned}
$$

(see [4]) where $P_{f}(w ; \zeta)$ is an analytic completion (as a function of $w$ ) of the Green's function $G_{f}(w, \zeta)$. That is,

$$
\begin{aligned}
& \log \left|\frac{w-w_{0}}{w^{* *}-w_{0}^{* *}}\right|+\Gamma_{f * *}\left(w^{* *}, w_{0}^{* *}\right)-\Gamma_{f}\left(w, w_{0}\right) \\
& =\operatorname{Re}\left\{\frac{\varepsilon}{\pi} \iint_{C} a(\omega) P_{f}^{\prime}(\omega ; w) P_{f}^{\prime}\left(\omega ; w_{0}\right) d m_{\omega}\right\}+o(\varepsilon) .
\end{aligned}
$$

Let $w \rightarrow w_{0}$; then

$$
\gamma_{f^{* *}}\left(w_{0}\right)-\gamma_{f}\left(w_{0}\right)=\operatorname{Re}\left\{\frac{\varepsilon}{\pi} \iint_{C} a(\omega)\left[P_{f}^{\prime}\left(\omega ; w_{0}\right)\right]^{2} d m_{\omega}\right\}+o(\varepsilon) .
$$

As in the proof of Theorem 1, the principal theorem of the variational method ([9]) implies that the extremal function $f$ for the problem $\min _{Q_{\Phi}(K, D)} \gamma_{f}\left(f\left(z_{0}\right)\right)$ satisfies the differential equation 


$$
\left[P_{f}\left(f(z) ; f\left(z_{0}\right)\right)\right]_{\bar{z}}=-\frac{K(z)-1}{K(z)+1} \overline{\left[P_{f}\left(f(z) ; f\left(z_{0}\right)\right)\right]_{z}} .
$$

As before, this is equivalent to the statement that $G_{f}\left(f(z), f\left(z_{0}\right)\right)=$ $\operatorname{Re} P_{f}\left(f(z) ; f\left(z_{0}\right)\right)$ is a solution of (7.3). Since

$$
G_{f}\left(f(z), f\left(z_{0}\right)\right)=\log \frac{1}{\left|f(z)-f\left(z_{0}\right)\right|}+\gamma_{f}\left(f\left(z_{0}\right)\right)+o(1) \quad \text { as } z \rightarrow z_{0},
$$

the proof that (7.4) has a limit as $z \rightarrow z_{0}$ is identical to the proof in Theorem 1.

Similarly, the extremal function $g$ for the problem $\max _{Q_{\Psi}(K, D)} \gamma_{f}\left(f\left(z_{0}\right)\right)$ satisfies the differential equation

$$
\left[P_{g}\left(g(z) ; g\left(z_{0}\right)\right)\right]_{\bar{z}}=\frac{K(z)-1}{K(z)+1} \overline{\left[P_{g}\left(g(z) ; g\left(z_{0}\right)\right)\right]_{z}},
$$

which implies that $G_{g}\left(g(z), g\left(z_{0}\right)\right)=\operatorname{Re} P_{g}\left(g(z) ; g\left(z_{0}\right)\right)$ is a solution of (7.6). The continuity of (7.7) follows as before.

If $\Omega$ is a domain that contains a neighborhood of $\infty$, then a harmonic Green's function for $\Omega$ with singularity at $\infty$ is a harmonic function $G(w)=G(w, \infty)$ in $\Omega$ that vanishes on $\partial \Omega$ and has the property that $\Gamma(w)=G(w)-\log |w|$ has a limit as $w \rightarrow \infty$. Note that $d=e^{-\Gamma(\infty)}$ is the transfinite diameter of $\Omega$. An entirely analogous development yields:

$\mathrm{Th}$ e or e m 4. Let $D$ be a domain that contains a neighborhood of $\infty$ and admits a harmonic Green's function with singularity at $\infty$. Suppose $K: D \rightarrow[1, \infty)$ is measurable, $\|K\|_{\infty}<\infty$, and $K(1 / z)$ satisfies $(2.4)$ at the origin. Then there exists a $K(z)$-q.c. mapping $f$ of $D$ such that $G_{f}(f(z))$, where $G_{f}$ is the harmonic Green's function of $f(D)$ with singularity at $\infty$, is a fundamental solution of (7.3) with zero boundary values, and $G_{f}(f(z))-$ $(1 / K(\infty)) \log |z|$ has a limit as $z \rightarrow \infty$. Similarly, there exists a $K(z)$-q.c. mapping $g$ of $D$ such that $G_{g}(g(z))$, where $G_{g}$ is the harmonic Green's function of $g(D)$ with singularity at $\infty$, is a fundamental solution of (7.6) with zero boundary values, and $G_{g}(g(z))-K(\infty) \log |z|$ has a limit as $z \rightarrow \infty$.

\section{A distortion theorem for $K(z)$-q.c. mappings of $D$}

Let $D$ be a domain bounded by a finite number of Jordan curves, and assume that $K: D \rightarrow[1, \infty)$ satisfies $(2.4)$ at fixed points $z_{1}, \ldots, z_{N} \in D$. As a measure of distortion for $f \in Q(K, D)$ we shall consider the functionals 


$$
\lambda[f]=\sum_{m, n=1}^{N} x_{m} x_{n}\left[\log \Phi\left(z_{m}, z_{n} ; K, f\right)-\Gamma_{f}\left(f\left(z_{m}\right), f\left(z_{n}\right)\right)\right]
$$

and

$$
\mu[f]=\sum_{m, n=1}^{N} x_{m} x_{n}\left[\log \Psi\left(z_{m}, z_{n} ; K, f\right)-\Gamma_{f}\left(f\left(z_{m}\right), f\left(z_{n}\right)\right)\right],
$$

where $\Gamma_{f}$ is the regular part of the harmonic Green's function for $f(D)$. We shall solve the problems

$$
\max _{Q(K, D)} \lambda \text { and } \min _{Q(K, D)} \mu:
$$

Theorem 5. Let $D$ be a domain bounded by a finite number of rectifiable Jordan curves, and assume that $K: D \rightarrow[1, \infty)$ is measurable, is essentially bounded, and satisfies (2.4) at fixed points $z_{1}, \ldots, z_{N^{N}} \in D$. If $f \in Q(K, D)$, then

$$
\begin{aligned}
\sum_{m, n=1}^{N} x_{m} x_{n}\left[\log \frac{\left|f\left(z_{m}\right)-f\left(z_{n}\right)\right|}{\left|z_{m}-z_{n}\right|^{\left[1 / K\left(z_{m}\right)+1 / K\left(z_{n}\right)\right] / 2}}-\Gamma_{f}\left(f\left(z_{m}\right), f\left(z_{n}\right)\right)\right. & \\
& \left.+s\left(z_{m}, z_{n} ; D\right)\right] \leqq 0
\end{aligned}
$$

and

$$
\begin{aligned}
\sum_{m, n=1}^{N} x_{m} x_{n}\left[\log \frac{\left|f\left(z_{m}\right)-f\left(z_{n}\right)\right|}{\left|z_{m}-z_{n}\right|^{\left[K\left(z_{m}\right)+K\left(z_{n}\right)\right] / 2}}-\Gamma_{f}\left(f\left(z_{m}\right), f\left(z_{n}\right)\right)\right. & \\
& \left.+\sigma\left(z_{m}, z_{n} ; D\right)\right] \geqq 0
\end{aligned}
$$

for any $x_{1}, \ldots, x_{N} \in \boldsymbol{R}$ satisfying $\sum_{n=1}^{N} x_{n}=0$. Here $\Gamma_{f}$ is the regular part of the harmonic Green's function for $f(D)$ and $s$ and $\sigma$ are the regular parts (7.4) and (7.7) of fundamental solutions to (7.3) and (7.6) in $D$ with zero boundary values.

Proof. The proof parallels the proof of Theorem 2. The existence of extremal functions for the problems (8.3) follows in the same fashion. Under the variation (5.13) one has ([4])

$$
\begin{aligned}
\lambda\left[f^{*}\right]-\lambda[f]= & -\sum_{\substack{m, n=1 \\
m \neq n}}^{N} x_{m} x_{n}\left[G_{f^{*}}\left(f^{*}\left(z_{m}\right), f^{*}\left(z_{n}\right)\right)-G_{f}\left(f\left(z_{m}\right), f\left(z_{n}\right)\right)\right] \\
& -\sum_{n=1}^{N} x_{n}^{2}\left[\gamma_{f^{*}}\left(f^{*}\left(z_{n}\right)\right)-\gamma_{f}\left(f\left(z_{n}\right)\right)\right] \\
= & -\operatorname{Re}\left\{\frac{\varepsilon}{\pi} \iint a(\omega)\left[\sum_{n}^{N} x_{n} P_{f}^{\prime}\left(\omega ; f\left(z_{n}\right)\right)\right]^{2} d m_{\omega}\right\}+o(\varepsilon) .
\end{aligned}
$$


Therefore the extremal function $f$ for the problem $\max _{Q(K, D)} \lambda$ has the property that $\sum_{n=1}^{N} x_{n} G_{f}\left(f(z), f\left(z_{n}\right)\right)$ satisfies the differential equation (7.3). Consequently,

$$
\sum_{n=1}^{N} x_{n}\left[G_{f}\left(f(z), f\left(z_{n}\right)\right)-S\left(z, z_{n} ; D\right)\right]=0
$$

for $z=z_{1}, \ldots, z_{N}$, just as in the proof of Theorem 2. The restriction of $\partial D$ to a rectifiable curve system was made to permit a boundary integration in verifying (8.6). Now (8.6) is equivalent to

$$
\sum_{n=1}^{N} x_{n}\left[\log \Phi\left(z_{m}, z_{n} ; K, f\right)-\Gamma_{f}\left(f\left(z_{m}\right), f\left(z_{n}\right)\right)+s\left(z_{m}, z_{n} ; D\right)\right]=0
$$

for all $m$, from which (8.4) follows.

The proof of $(8.5)$ is similar.

\section{Fundamental solutions of the second kind}

The harmonic Neumann's function $N(w, \zeta)$ of a domain $\Omega$ bounded by a finite number of Jordan curves with a continuously turning tangent has the properties that, as a function of $w$,

(1) $N(w, \zeta)$ is harmonic in $\Omega-\{\zeta\}$,

(2) $N(w, \zeta)+\log |w-\zeta|$ is harmonic at $\zeta$,

(3) $\frac{\partial N(w, \zeta)}{\partial n_{w}}=\frac{2 \pi}{L}$, where $L$ is the length of $\partial \Omega$, and

(4) $\int_{\partial \Omega} N(w, \zeta) d s_{w}=0$

It is a consequence of Green's theorem that $N$ is symmetric: $N(w, \zeta)=$ $N(\zeta, w)$. Let $\Pi(w ; \zeta)$ be an (multivalued) analytic completion as a function of $w$ (so that $N=\operatorname{Re} \Pi$ ).

We shall also use the related functions:

$$
\begin{gathered}
\Pi(w ; \zeta, \eta)=\Pi(w ; \zeta)-\Pi(w ; \eta) \\
N(w, \zeta, \eta)=\operatorname{Re} \Pi(w ; \zeta, \eta)=N(w, \zeta)-N(w, \eta), \\
\Pi(w, \omega ; \zeta, \eta)=\Pi(w ; \zeta, \eta)-\Pi(\omega ; \zeta, \eta) \\
N(w, \omega, \zeta, \eta)=\operatorname{Re} \Pi(w, \omega ; \zeta, \eta)=N(w, \zeta, \eta)-N(\omega, \zeta, \eta) .
\end{gathered}
$$

It is elementary to verify the addition formulae: 


$$
\begin{aligned}
\Pi(w, \omega ; \zeta, \eta) & =\Pi(w, \omega ; \zeta, \xi)+\Pi(w, \omega ; \xi, \eta) \\
\Pi^{\prime}(w ; \zeta, \eta) & =\Pi^{\prime}(w ; \zeta, \xi)+\Pi^{\prime}(w ; \xi, \eta)
\end{aligned}
$$

where the derivative will always be with respect to the first variable. The symmetry relation

$$
N(w, \omega, \zeta, \eta)=N(\zeta, \eta, w, \omega)
$$

follows from that of $N(w, \zeta)$. As a consequence, one has the limit values

$$
N(w, \omega, \zeta, \zeta)=N(\zeta, \zeta, w, \omega)=0 .
$$

The function (9.2) has boundary values of type $N$ in the sense of [4]. That is, under an interior variation of the form

$$
w^{*}=w+\frac{\varepsilon e^{i \vartheta}}{w-w_{0}},
$$

the representation

$$
\begin{aligned}
& N^{*}\left(w^{*}, \omega^{*}, \zeta^{*}, \eta^{*}\right)-N(w, \omega, \zeta, \eta) \\
& =\frac{1}{2 \pi} \int\left[N(\xi, w, \omega) \frac{\partial D(\xi, \zeta, \eta)}{\partial n_{\xi}}-D(\xi, \zeta, \eta) \frac{\partial N(\xi, w, \omega)}{\partial n_{\xi}}\right] d s_{\xi}
\end{aligned}
$$

where the integral is extended over $\partial \Omega+\left\{\left|\xi-w_{0}\right|=\sqrt{\varepsilon}\right\}$, and

$$
D(w, \zeta, \eta)=N^{*}\left(w^{*}(w), \zeta^{*}(\zeta), \eta^{*}(\eta)\right)-N(w, \zeta, \eta),
$$

has the property that the integral over $\partial \Omega$ vanishes. By evaluating the integral over the small circle $\left|\xi-w_{0}\right|=\sqrt{\varepsilon}$, as in [4], one obtains the variational formula

$$
\begin{aligned}
& N^{*}\left(w^{*}, \omega^{*}, \zeta^{*}, \eta^{*}\right)-N(w, \omega, \zeta, \eta) \\
& =\operatorname{Re}\left\{\varepsilon e^{i \vartheta} \Pi^{\prime}\left(w_{0} ; w, \omega\right) \Pi^{\prime}\left(w_{0} ; \zeta, \eta\right)\right\}+o(\varepsilon) .
\end{aligned}
$$

We wish to extend the variational formula (9.10) to more general domains $\Omega$. The key will be to use the fact that $F(w ; \zeta, \eta)=e^{\Pi(w ; \zeta, \eta)}$ is single valued and maps $\Omega$ conformally onto a domain bounded by radial slits. It is normalized so that $w=\zeta$ corresponds to $\infty$ and $w=\eta$ to the origin.

Any domain $\hat{\Omega}$ bounded by finitely many nondegenerate continua admits radial slit mappings $\hat{F}(w ; \zeta, \eta)$ taking $\zeta$ to $\infty$ and $\eta$ to 0 . The mappings are determined by this normalization up to a multiplicative constant, so that for smoothly bounded domains

$$
\hat{\Pi}(w ; \zeta, \eta)=\log \hat{F}(w ; \zeta, \eta)
$$


differs from $\Pi(w ; \zeta, \eta)$ by at most a constant (depending on $\zeta$ and $\eta$ ). However,

$$
\hat{\Pi}(w, \omega ; \zeta, \eta)=\hat{\Pi}(w ; \zeta, \eta)-\hat{\Pi}(\omega ; \zeta, \eta)
$$

and $\hat{\Pi}^{\prime}(w ; \zeta, \eta)$; are uniquely determined and agree with their conterparts in case $\partial \hat{\Omega}$ is smooth. At the same time, $\hat{N}(w, \omega, \zeta, \eta)=$ $\operatorname{Re} \hat{\Pi}(w, \omega ; \zeta, \eta)$ is uniquely determined, and

$$
\begin{gathered}
\hat{N}(w, \zeta, \eta)=\operatorname{Re} \hat{\Pi}(w ; \zeta, \eta)=\log |\hat{F}(w ; \zeta, \eta)|, \\
\hat{v}(w, \zeta, \eta)=\hat{N}(w, \zeta, \eta)+\log \left|\frac{w-\zeta}{w-\eta}\right|
\end{gathered}
$$

are determined up to an additive constant (depending on $\zeta$ and $\eta$ ).

By Carathéodory's theorem on domain convergence, the functions $\hat{\Pi}(w, \omega ; \zeta, \eta)$ and $\hat{\Pi}^{\prime}(w, \zeta, \eta)$ are the limits of functions $\Pi_{n}(w, \omega ; \zeta, \eta)$ and $\Pi_{n}^{\prime}(w ; \zeta, \eta)$ corresponding to mappings $F_{n}(w ; \zeta, \eta)$ of a smoothly bounded exhaustion $\left\{\Omega_{n}\right\}$ of $\hat{\Omega}$. Consequently, the variational formula (9.10) carries over to the functionals $\hat{N}$ and $\hat{\Pi}^{\prime}$ for $\hat{\Omega}$. For the same reason, the identities $(9.5)-(9.8)$ also carry over in terms of $\hat{\Pi}$ and $\hat{N}$.

Now fix points $w_{1}, \ldots, w_{M}$ in a domain $\hat{\Omega}$ bounded by a finite number of nondegenerate continua, and let $x_{1}, \ldots, x_{M} \in \boldsymbol{R}$ with $\sum_{m=1}^{M} x_{m}=0$. Substitute $w=w_{m}$ into the extended form of (9.10) and sum:

$$
\begin{aligned}
& \sum_{m=1}^{M} x_{m}\left[\hat{N} *\left(w_{m}^{*}, \zeta^{*}, \eta^{*}\right)-\hat{N}\left(w_{m}, \zeta, \eta\right)\right] \\
= & \operatorname{Re}\left\{\varepsilon e^{i \vartheta} \hat{\Pi}^{\prime}\left(w_{0} ; \zeta, \eta\right) \sum_{m=1}^{M} x_{m} \hat{\Pi}^{\prime}\left(w_{0} ; w_{m}, \omega\right)\right\}+o(\varepsilon) .
\end{aligned}
$$

It follows from the addition formula (9.6) that the last sum in (9.15) is independent of $\omega$. Therefore, we may as well let $\omega=\eta$. Now let $\zeta=w_{n}$ and sum; then

$$
\begin{aligned}
& \sum_{m, n=1}^{M} x_{m} x_{n}\left[\log \frac{\left|w_{m}-w_{n}\right|}{\left|w_{m}^{*}-w_{n}^{*}\right|}+\hat{v}^{*}\left(w_{m}^{*}, w_{n}^{*}, \eta^{*}\right)-\hat{v}\left(w_{m}, w_{n}, \eta\right)\right] \\
= & \operatorname{Re}\left\{\varepsilon e^{i \vartheta}\left[\sum_{m=1}^{M} x_{m} \hat{\Pi}^{\prime}\left(w_{0} ; w_{m}, \eta\right)\right]^{2}\right\}+o(\varepsilon),
\end{aligned}
$$

where for $m=n$ the quotient $\left|\left(w_{m}-w_{n}\right) /\left(w_{m}^{*}-w_{n}^{*}\right)\right|$ is $1 /\left|w^{* \prime}\left(w_{n}\right)\right|$. Since $\sum_{m=1}^{M} x_{m}=0$, the expressions in (9.15) and (9.16) are independent of which mapping $\hat{F}$ is used to generate $\hat{N}, \hat{v}$, and $\hat{\Pi}^{\prime}$. In addition, because of (9.5) and (9.6), both sides of (9.16) are independent of $\eta$, and we may write the right side as 


$$
\operatorname{Re}\left\{\varepsilon e^{i \vartheta}\left[\sum_{m=1}^{M-1} x_{m} \hat{\Pi}^{\prime}\left(w_{0} ; w_{m}, w_{M}\right)\right]^{2}\right\}+o(\varepsilon) .
$$

Assume now that $D$ is a domain bounded by finitely many nondegenerate continua and that $K: D \rightarrow[1, \infty)$ satisfies $(2.4)$ at fixed points $z_{1}, \ldots, z_{M} \in D$. For $f \in Q(K, D)$, define

$$
\chi[f]=\sum_{m, n=1}^{M} x_{m} x_{n}\left[\log \Phi\left(z_{m}, z_{n} ; K, f\right)-\hat{v}_{f}\left(f\left(z_{m}\right), f\left(z_{n}\right), \eta\right)\right]
$$

and

$$
x[f]=\sum_{m, n=1}^{M} x_{m} x_{n}\left[\log \Psi\left(z_{m}, z_{n} ; K, f\right)-\hat{v}_{f}\left(f\left(z_{m}\right), f\left(z_{n}\right), \eta\right)\right],
$$

where $\hat{v}_{f}$ is the regular part (9.14) of any function (9.13) for $\hat{\Omega}=f(D)$. Since $\sum_{m=1}^{M} x_{m}=0, \quad \chi$ and $x$ are independent of which normalization is used in the mapping $\hat{F}$. In addition, because of (9.5) both functionals are independent of $\eta$.

We shall consider the problems

$$
\max _{Q(K, D)} \chi \text { and } \min _{Q(K, D)} \varkappa .
$$

Existence of extremal functions for these problems follows as before (cf. Theorems 2 and 5). Using (9.16), we find that an extremal function $f$ for the problem $\max _{Q_{(K, D)}} \chi$ satisfies

$$
\chi\left[f^{*}\right]=\chi[f]-\operatorname{Re}\left\{\varepsilon e^{i \vartheta}\left[\sum_{m=1}^{M-1} x_{m} \hat{\Pi}^{\prime}\left(w_{0} ; f\left(z_{m}\right), f\left(z_{M}\right)\right)\right]^{2}\right\}+o(\varepsilon)
$$

under a variation of the form (9.9). A similar formula arises from the variation (5.13). Consequently, the principal theorem of the variational method (see [9, Corollary 13.3]) leads to the differential equation

$$
H_{\bar{z}}=-\frac{K-1}{K+1} \bar{H}_{z}
$$

for

$$
H(z)=\sum_{m=1}^{M} x_{m} \hat{\Pi}_{f}\left(f(z), f\left(z_{m}\right), \eta\right)=\sum_{m=1}^{M-1} x_{m} \hat{\Pi}_{f}\left(f(z), f\left(z_{m}\right), f\left(z_{M}\right)\right) .
$$

Similarly, an extremal function $g$ for the problem $\min _{Q(K, D)} x$ satisfies

$$
J_{\bar{z}}=\frac{K-1}{K+1} \bar{J}_{z}
$$

where

$$
J(z)=\sum_{m=1}^{M} x_{m} \hat{\Pi}_{g}\left(g(z), g\left(z_{m}\right), \eta\right)=\sum_{m=1}^{M-1} x_{m} \hat{\Pi}_{g}\left(g(z), g\left(z_{m}\right), g\left(z_{M}\right)\right) .
$$


It also follows, as in the proof of Theorem 1, that $\operatorname{Re} H$ has singularities of the form $\left(x_{m} / K\left(z_{m}\right)\right) \log \left(1 /\left|z-z_{m}\right|\right)$ and $\operatorname{Re} J$ has singularities of the form $x_{m} K\left(z_{m}\right) \log \left(1 /\left|z-z_{m}\right|\right), m=1, \ldots, M$.

The special case $x_{1}=1, x_{2}=-1, M=2$ of the preceding discussion is already of interest. Let us summarize it using the notation

$$
\begin{aligned}
\tilde{S}\left(z, z_{1}, z_{2} ; D\right) & +i \tilde{T}\left(z, z_{1}, z_{2} ; D\right)=\hat{\Pi}_{f}\left(f(z) ; f\left(z_{1}\right), f\left(z_{2}\right)\right) \\
& =\log \hat{F}_{f}\left(f(z) ; f\left(z_{1}\right), f\left(z_{2}\right)\right)=\log \tilde{F}\left(z ; z_{1}, z_{2}\right), \\
\tilde{\Sigma}\left(z, z_{1}, z_{2} ; D\right) & +i \tilde{\tilde{I}}\left(z, z_{1}, z_{2} ; D\right)=\hat{\Pi}_{g}\left(g(z) ; g\left(z_{1}\right), g\left(z_{2}\right)\right) \\
& =\log \hat{F}_{g}\left(g(z) ; g\left(z_{1}\right), g\left(z_{2}\right)\right)=\log \mathfrak{S}\left(z ; z_{1}, z_{2}\right) .
\end{aligned}
$$

Th e o r e $\mathrm{m}$ 6. Let $D$ be a domain bounded by a finite number of nondegenerate continua, and assume that $K: D \rightarrow[1, \infty)$ is measurable, is essentially bounded, and satisfies (2.4) at $z_{1}, z_{2} \in D$. Then there exists a $K(z)$-q.c. mapping $\mathfrak{F}\left(z ; z_{1}, z_{2}\right)$ of $D$ onto the extended plane with radial slits, such that

$$
\tilde{S}\left(z, z_{1}, z_{2} ; D\right)=\log \left|\mathfrak{F}\left(z ; z_{1}, z_{2}\right)\right|
$$

is a (weak) fundamental solution of the differential equation

$$
\operatorname{div}(K \operatorname{grad} U)=0
$$

in $D$ with singularities at $z_{1}$ and $z_{2}$. Furthermore,

$\tilde{s}\left(z, z_{1}, z_{2} ; D\right)=\tilde{S}\left(z, z_{1}, z_{2} ; D\right)+\frac{1}{K\left(z_{1}\right)} \log \left|z-z_{1}\right|-\frac{1}{K\left(z_{2}\right)} \log \left|z-z_{2}\right|$

has a limit as $z \rightarrow z_{1}$ and as $z \rightarrow z_{2}$. Similarly, there exists a $K(z)$-q.c.

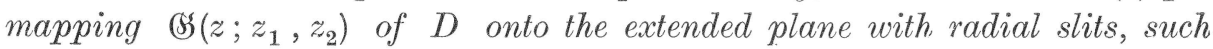
that

$$
\tilde{\Sigma}\left(z, z_{1}, z_{2} ; D\right)=\log \left|\circlearrowleft\left(z ; z_{1}, z_{2}\right)\right|
$$

is a (weak) fundamental solution of the differential equation

$$
\operatorname{div}((1 / K) \operatorname{grad} U)=0
$$

in $D$ with singularities at $z_{1}$ and $z_{2}$. Furthermore,

$$
\begin{aligned}
& \tilde{\sigma}\left(z, z_{1}, z_{2} ; D\right) \\
& =\tilde{\Sigma}\left(z, z_{1}, z_{2} ; D\right)+K\left(z_{1}\right) \log \left|z-z_{1}\right|-K\left(z_{2}\right) \log \left|z-z_{2}\right|
\end{aligned}
$$

has a limit as $z \rightarrow z_{1}$ and as $z \rightarrow z_{2}$.

The functions $\tilde{S}$ and $\Sigma$ are fundamental solutions of the second kind 
in the sense that their conjugates $\tilde{T}$ and $\tilde{I}$ are constant on each boundary component of $D$.

We may now solve the problems (9.19) in terms of the fundamental solutions $\tilde{S}$ and $\tilde{\Sigma}$. We omit the rest of the proof because of its similarity to the proof of Theorem 5 .

$\mathrm{T} \mathrm{h}$ e o $\mathrm{r}$ e $\mathrm{m}$ 7. Let $D$ be a domain bounded by a finite number of Jordan curves with a continuously turning tangent. Assume that $K: D \rightarrow[1, \infty)$ is measurable, is essentially bounded, and satisfies (2.4) at $z_{1}, \ldots, z_{M} \in D$. If $f \in Q(K, D)$ then

$$
\begin{aligned}
& \sum_{m, n=1}^{M} x_{m} x_{n}\left[\log \frac{\left|f\left(z_{m}\right)-f\left(z_{n}\right)\right|}{\left|z_{m}-z_{n}\right|^{\left[1 / K\left(z_{m}\right)+1 / K\left(z_{n}\right)\right] / 2}}-\hat{v}_{f}\left(f\left(z_{m}\right), f\left(z_{n}\right), \eta\right)\right] \\
& +\sum_{m, n=1}^{M-1} x_{m} x_{n}\left[\tilde{s}\left(z_{m}, z_{n}, z_{M} ; D\right)-\tilde{s}\left(z_{M}, z_{n}, z_{M} ; D\right)\right] \leqq 0
\end{aligned}
$$

and

$$
\begin{aligned}
& \sum_{m, n=1}^{M} x_{m} x_{n}\left[\log \frac{\left|f\left(z_{m}\right)-f\left(z_{n}\right)\right|}{\left|z_{m}-z_{n}\right|^{\left[K\left(z_{m}\right)+K\left(z_{n}\right)\right] / 2}}-\hat{v}_{f}\left(f\left(z_{m}\right), f\left(z_{n}\right), \eta\right)\right] \\
& +\sum_{m, n=1}^{M-1} x_{m} x_{n}\left[\tilde{\sigma}\left(z_{m}, z_{n}, z_{M} ; D\right)-\tilde{\sigma}\left(z_{M}, z_{n}, z_{M} ; D\right)\right] \geqq 0
\end{aligned}
$$

for any $x_{1}, \ldots, x_{M} \in \boldsymbol{R}$ satisfying $\sum_{m=1}^{M} x_{m}=0$. Here $\hat{v}_{f}$ is the regular part (9.14) of any function $\hat{N}_{f}(w, \zeta, \eta)$ for $f(D)$ and $\hat{s}$ and $\hat{\sigma}$ are the regular parts (9.22) and (9.24) of fundamental solutions of the second kind for (9.21) and (9.23).

\section{Mapping theorems}

Many extremum problems lead to existence theorems for canonical mappings by means of $K(z)$-q.c. mappings. A characteristic example is already provided by Theorem 6 . In this section we add two further results of this nature.

Let $D$ be a domain which possesses a harmonic Green's function, and assume that $K: D \rightarrow[1, \infty)$ satisfies $(2.4)$ at $z_{0} \in D$. Then, on the basis of Theorem 3, we can assert the existence of a $K(z)$-q.c. mapping $f$ of $D$ onto a domain $\Omega=f(D)$ such that the combined function

$$
U(z)=G_{i}\left(f(z), f\left(z_{0}\right)\right),
$$

where $G_{f}$ is the harmonic Green's function of $\Omega$, satisfies the differential equation

$$
\operatorname{div}(K \operatorname{grad} U)=0
$$


Moreover, if $P_{f}\left(w ; w_{0}\right)$ is any (multivalued) analytic completion of $G_{f}\left(w, w_{0}\right)$ (as a function of $w$ ), then the function.

$$
\mathfrak{A}(z)=P_{f}\left(f(z) ; f\left(z_{0}\right)\right)=U(z)+i V(z)
$$

generates a function $V$, conjugate to $U$ by the generalized CauchyRiemann equations, which can be combined into

$$
\mathfrak{A}_{\bar{z}}=-\frac{K-1}{K+1} \overline{\mathfrak{A}}_{z} .
$$

If $D$ is simply connected, then so is $\Omega$, and $\exp \left\{-P_{f}\left(w ; w_{0}\right)\right\}$ is single valued and maps $\Omega$ conformally onto the unit disk with $w_{0}$ corresponding to the origin. Consequently, $\exp \{-\mathfrak{A}\}$ is univalent and maps $D$ onto the unit disk with $z_{0}$ corresponding to the origin.

In the same manner, the $K(z)$-q.c. mapping $g$ of Theorem 3 leads to a solution

$$
\mathfrak{B}(z)=P_{g}\left(g(z) ; g\left(z_{0}\right)\right)
$$

of the generalized Cauchy-Riemann equations

$$
\mathfrak{B}_{\bar{z}}=\frac{K-1}{K+1} \overline{\mathfrak{B}_{z}}
$$

such that $\exp \{-\mathfrak{B}\}$ has similar mapping properties. We thus have the following generalization of the Riemann mapping theorem:

T h e o r e $\mathrm{m}$ 8. Let $D$ be simply connected and have at least two boundary points. Assume that $K: D \rightarrow[1, \infty)$ is measurable, is essentially bounded, and satisfies (2.4) at $z_{0} \in D$. Then there exist functions $\mathfrak{A}$ and $\mathfrak{B}$ that satisfy the generalized Cauchy-Riemann equations (10.1) and (10.2), respectively, such that $\exp \{-\mathfrak{U}\}$ and $\exp \{-\mathfrak{B}\}$ are univalent mappings of $D$ onto the unit disk with $z_{0}$ corresponding to the origin.

A similar result can be obtained in the case of a multiply connected domain $D$. We introduce for this purpose the modified harmonic Green's function $\tilde{G}(w, \omega)$ of a domain $\Omega$ with proper continua $C_{0}, C_{1}, \ldots, C_{n}$ as boundaries. It is defined as follows:

(1) $\tilde{G}(w, \omega)$ is harmonic in $\Omega-\{\omega\}$ (as a function of $w$ ).

(2) $\tilde{G}(w, \omega)+\log |w-\omega|$ is harmonic at $\omega$.

(3) $\tilde{G}(w, \omega)$ is constant on all $C_{v}$; in particular, $\tilde{G}(w, \omega)=0$ on $C_{0}$.

(4) $\int \frac{\partial \tilde{G}(w, \omega)}{\partial n_{w}} d s_{w}=0$ on all smooth curves homotopic to $C_{v}$ in $\Omega-\{\omega\}, v=1, \ldots, n$. 
(5) $\int \frac{\partial \tilde{G}(w, \omega)}{\partial n_{w}} d s_{w}=2 \pi$ on all smooth curves homotopic to $C_{0}$ in $\Omega-\{\omega\}$.

This modified Green's function can be constructed directly from the ordinary Green's function of $\Omega$ and the harmonic measures of $C_{1}, \ldots, C_{n}$.

If $\tilde{P}(w ; \omega)$ is an analytic completion of $\tilde{G}(w, \omega)$ (as a function of $w$ ), then $\exp \{-\tilde{P}(w ; \omega)\}$ maps $\Omega$ onto the unit disk slit along concentric circular arcs, such that $C_{0}$ corresponds to the unit circumference and $\omega$ to the origin.

The modified Green's function also satisfies the condition $N$ of [4], used before, and thus has, under a variation (9.9) of the domain, the asymptotic behavior

$$
\tilde{G}^{*}\left(w^{*}, \omega^{*}\right)=\tilde{G}(w, \omega)+\operatorname{Re}\left\{\varepsilon e^{i \vartheta} \tilde{P}^{\prime}\left(w_{0} ; w\right) \tilde{P}^{\prime}\left(w_{0} ; \omega\right)\right\}+o(\varepsilon) .
$$

Using again the extremal problems for $\tilde{G}_{f}\left(f(z), f\left(z_{0}\right)\right)$ which led to Theorem 3, we arrive at the following:

$\mathrm{T}$ h e o $\mathrm{r}$ e $\mathrm{m}$ 9. Let $D$ be a domain bounded by a finite number of proper continua. Assume that $K: D \rightarrow[1, \infty)$ is measurable, is essentially bounded, and satisfies (2.4) at $z_{0} \in D$. Then there exist functions $\mathfrak{A}$ and $\mathfrak{B}$ that satisfy the generalized Cauchy-Riemann equations (10.1) and (10.2), respectively, such that $\exp \{-\mathfrak{A}\}$ and $\exp \{-\mathfrak{B}\}$ are univalent mappings of $D$ onto the unit disk slit along concentric circular arcs, with a prescribed boundary corresponding to the unit circumference and $z_{0}$ to the origin.

Many analogous canonical mapping theorems can be established in the same way. The method always relies on finding an extremum problem for an appropriate functional with a convenient variational formula.

\section{References}

[1] KÜHNAU, R.: Verzerrungssätze und Koeffizientenbedingungen vom Grunsky schen Typ für quasikonforme Abbildungen. - Math. Nachr. 48, 1971, 77-105.

[2] LEHTO, O., and K. I. VIRTANEN: Quasiconformal mappings in the plane. Springer-Verlag, Berlin-Heidelberg-New York, 1973.

[3] RENEIT, H.: Modifizierung und Erweiterung einer Schifferschen Variationsmethode für quasikonforme Abbildungen. - Math. Nachr. 55, 1973, $353-379$.

[4] SCHIFFER, M.: Hadamard's formula and variation of domain-functions. - Amer. J. Math. 68, 1946, 417-448.

[5] - - Fredholm eigen values of multiply-connected domains. - Pacific J. Math. 9, 1959, 211-269.

[6] -»- A variational method for univalent quasiconformal mappings. - Duke Math. J. 33, 1966, 395-412. 
[7] SCHIFFER, M., and G. SCHOBER: An extremal problem for the Fredholm eigenvalues. - Arch. Rational Mech. Anal. 44, 1971, 83-92, and 46, 1972, 394.

[8] - - A distortion theorem for quasiconformal mappings. - Advances in complex function theory, Lecture Notes in Mathematics 505, SpringerVerlag, Berlin-Heidelberg-New York, 1976.

[9] Schober, G.: Univalent functions - Selected topics. - Lecture Notes in Mathematics 478, Springer-Verlag, Berlin-Heidelberg-New York, 1975.

[10] STREBEI, K.: Ein Konvergenzsatz für Folgen quasikonformer Abbildungen. Comment. Math. Helv. 44, 1969, 469-475.

Stanford University

Department of Mathematics

Stanford, California 94305

USA

Received 27 July 1975
Indiana University

Department of Mathematics

Bloomington, Indiana 47401

USA 